\title{
Gravitational theories with topological invariant
}

\begin{abstract}
This paper provides a brief review of the gravitational theories which have topological classes, such as the Chern-Simmons, BF Theory and Chern-Weil theory. For quantization of the Yang-Mills field theory, gauge symmetries and corresponding conserved charges play an essential role. These properties, symmetries and conserved charges, correspond to a structural group and topological classes of a principal bundle in differential geometry. Gravitational theories which have topological classes with respect to the structural group of the principal bundle are possible starting point of the still-unknown quantum gravitational theory.
\end{abstract}

Keywords: Chern-Weil field theory, gauge symmetries, conserved charges, topological classes, structural group, gravitational theories
Volume 2 Issue 4 - 2018

\section{Yoshimasa Kurihara}

The High Energy Accelerator Organization (KEK), Japan

Correspondence: Yoshimasa Kurihara, The High Energy Accelerator Organization (KEK), Oho I-I,Tsukuba, lbaraki 305-080I, Japan, Tel +8I29 8796088 ,

EmailYoshimasa.kurihara@kek.jp

Received: July 30, 2018 | Published: August 16, 2018

\section{Classical general relativity}

A four-dimensional pseudo-Riemannian manifold $(\mathcal{M}, g)$ with $G L(n)$ symmetry is introduced as a model of the universe. General relativity is constructed in $\mathcal{M}$ as follows: The line element is defined using the metric tensor as $d s^{2}:=g_{\mu_{1} \mu_{2}}(x) d x^{\mu_{1}} \otimes d x^{\mu_{2}}$. (The Einstein convention for repeated indices is used throughout this report.) It is always possible to find a frame of reference whose affine connection as $\Gamma_{\mu \nu}^{\rho}(p)=0$ at any point $p \in \mathcal{M}$. A local manifold with a vanishing affine connection displays Poincaré symmetry $I S O(n)=S O(n) \ltimes T(n)$, where $T(n)$ is a group of $n$-dimensional translations. We introduce a $n$-dimensional Riemannian manifold $\left(M_{p}, \eta\right)$ with vanishing affine connection at $p \in \mathcal{M}$ and a bundle $M:=\bigcup_{p} M_{p}$. The diffeomorphism $\operatorname{map} \mathcal{E}: \mathcal{M} \rightarrow M: \mathbf{g} \mapsto \eta$ is represented using standard coordinates on any chart of $\mathcal{M}$ as

$$
\eta^{a b}=\mathcal{E}_{\mu_{1}}^{a}\left(x^{\mu}\right) \mathcal{E}_{\mu_{2}}^{b}\left(x^{\mu}\right) g^{\mu_{1} \mu_{2}}\left(x^{\mu}\right) \text {. }
$$

The function $\mathcal{E}_{\mu}^{a}\left(x^{\mu}\right)=\left[\mathcal{E}\left(x^{\mu}\right)\right]_{\mu}^{a}$ is referred to as a vierbein. The spin connection $\mathfrak{w} \in \mathfrak{s o}(1,3)$ is introduced as a Lie-algebra-valued one-form, and is referred to as the spin form. It can be represented in standard coordinates as $\mathfrak{w}^{a b}=\omega_{\mu c}^{a} \eta^{c b} d x^{\mu}$, and is antisymmetric, i.e., $\mathfrak{w}^{a b}=-\mathfrak{w}^{b a}$.

The Einstein-Hilbert gravitational action can be expressed as

$$
\mathcal{I}=\frac{1}{2} \int_{\Sigma_{n}}\left(\varepsilon_{a_{1} \cdots a_{n}} \mathfrak{R}^{a_{1} a_{2}} \wedge \mathfrak{e}^{a_{3}} \wedge \cdots \wedge \mathfrak{e}^{a_{n}}\right),
$$

where $\mathfrak{R}^{a b}:=d \mathfrak{w}^{a b}+\mathfrak{w}^{a}{ }_{c} \wedge \mathfrak{w}^{c b}$ is a curvature two-form. Theories which have topological (characteristic) classes are referred to as the topological theory in this article. We note that the topological theory does not means the so-called "topological field theory", which has the metric independent correlation functions, and thus, it does not exhibit any dynamics. Three examples of the topological theories are reviewed.

\section{Topological theories}

On a $n$-dimensional Riemannian manifold $P(\mathcal{M}, G)$, a principal bundle $P(\mathcal{M}, G)$ is introduced, where $G$ is the structural Lie-group. The connection one-form $\mathfrak{A}$ and the corresponding curvature twoform $\mathfrak{F}=d \mathfrak{A}+\mathfrak{A} \wedge \mathfrak{A}$ are equipped on the base manifold $M . \mathfrak{A}$ is a
Lie-algebra valued one-form $\mathfrak{A} \in \mathfrak{g}$, where $\mathfrak{g}$ is a Lie-algebra of the structural group $G$.

\section{Chern-Simons topological theory}

In 1988, Witten shows that general relativity in the $(1+2)-$ dimensional space can be considered as a Chern-Simons topological theory. ${ }^{1}$ In this theory, we employ a principal bundle consists of the base manifold $\mathcal{M}$ with $(1+2)$ space-time dimension and the structural group ISO $(1,2)$. The connection one-form and curvature two-form can be respectively introduced as

$$
\begin{aligned}
& \mathfrak{A}_{C S}:=\left\{J_{a b}, P_{c}\right\} \times\left\{\mathfrak{w}^{a b}, \mathfrak{e}^{c}\right\}=J_{a b} \mathfrak{w}^{a b}+P_{c} \mathfrak{e}^{c}, \\
& \mathfrak{F}_{C S}:=d \mathfrak{A}_{C S}+\mathfrak{A}_{C S} \wedge \mathfrak{A}_{C S}=J_{a b} \mathfrak{R}^{a b}+P_{c} \mathfrak{T}^{c},
\end{aligned}
$$

where $J_{a b}$ and $P_{a}$ are respectively the Lie-algebra of a rotation and a translation, and $\mathfrak{T}^{a}:=d \mathfrak{e}^{a}+\mathfrak{w}_{b}^{a} \wedge \mathfrak{e}^{b}$ is a torsion two-form. The Chern-Simons action is thus obtained as

$$
\begin{gathered}
\mathcal{I}_{C S}:=\frac{1}{4} \int_{\Sigma_{4}} \mathfrak{F}_{C S} \wedge \mathfrak{F}_{C S}, \\
=\frac{1}{2} \int_{\Sigma_{4}} \operatorname{Tr}\left[\mathfrak{A}_{C S} \wedge d \mathfrak{A}_{C S}+\frac{2}{3} \mathfrak{A}_{C S} \wedge \mathfrak{A}_{C S} \wedge \mathfrak{A}_{C S}\right], \\
=\frac{1}{2} \int_{\Sigma_{4}} d\left(\varepsilon_{a b c} \mathfrak{e}^{a} \wedge \mathfrak{R}^{b c}\right), \\
=\frac{1}{2} \int_{\partial \Sigma_{4}=\Sigma_{3}}\left(\varepsilon_{a b c} \mathfrak{e}^{a} \wedge \mathfrak{R}^{b c}\right),
\end{gathered}
$$

where $\Sigma_{4}$ is an appropriate simply connected and orientable four-dimensional manifold in which a three-dimensional manifold $\Sigma_{3} \subset M$ is immersed. This is nothing other than the EinsteinHilbert action without the cosmological term, and thus, it is shown that general relativity can be constructed as the Chern-Simons topological theory in three-dimension. This coincidence between the Chern-Simons and Einstein-Hilbert actions is rather accidental only in the three dimensional space. , $^{2,3}$

Quantization of the theory can be performed using the canonical commutation-relations on $\mathfrak{e}$ and $\mathfrak{w}$. Whereas the Chern-Simins quantum gravity in three-dimensional space does not have any dynamical degree, this is not owing to a topological aspect of the theory. From a simple counting of the degree of freedom (DOF), one 
can understand that the dynamical DOF in three-dimensional general relativity is zero at the classical level. Detailed summary of ChernSimons (super) gravity can be found in a textbook. ${ }^{4}$

\section{BF topological theory}

In 1977, the BF topological theory is introduced at first by Plebański ${ }^{5}$, while the term "BF theory" did not exist yet at that time. In 1989 , Horowitz first treated general relativity of the BF theory as the topological theory in general $n_{\text {-dimensional space-time. }}{ }^{6}$ Review articles of the $\mathrm{BF}$ topological theory can be found. ${ }^{7,8}$

In the $\mathrm{BF}$ theory, the base manifold $M$ is chosen as a fourdimensional Riemannian manifold and the structural group is taken as $S O(1,3)$ or $S O(4)$. The connection one-form and curvature twoform can be respectively taken as the spin form $\mathfrak{w}^{a b}$ and curvature form $\mathfrak{R}^{a b}$. In addition, new Lie-algebra valued two-form $\mathfrak{B}^{a b}$ is introduced, and an action in the BF theory is defined as

$$
\tilde{\mathcal{I}}_{B F}:=\frac{1}{2} \int_{\Sigma_{4}} \varepsilon_{a b c d} \mathfrak{B}^{a b} \wedge \mathfrak{R}^{c d},
$$

where $\Sigma_{4}$ is an appropriate simply connected and orientable fourdimensional manifold. This action cannot be simply recognized as the topological action of the structural group $S O(1,3)$, because the form $\mathfrak{B}^{a b}$ does not belong to the principal bundle in general. Instead, the form $\mathfrak{B}^{a b}$ must be understood as a connection form on a 2 -bundle, and it forms principal 2-bundle in the higher-gauge theory. ${ }^{9}$ Whereas the action $\tilde{\mathcal{I}}_{B F}$ can be topological by means of the 2 -gauge theory, it is not coincide with the Einstein-Hilbert action. To convert the BF topological theory to a gravitational theory, additional constraints ${ }^{5,10}$ must be implement as, e.g., a Lagrange multiplier term such as

$$
\mathcal{I}_{B F}:=\frac{1}{2} \int_{\Sigma_{4}}\left(\varepsilon_{a b c d} \mathfrak{B}^{a b} \wedge \mathfrak{R}^{c d}-\frac{1}{2} \phi_{a b c d} \mathfrak{B}^{a b} \wedge \mathfrak{B}^{c d}\right),
$$

where $\phi_{a b c d}$ is a Lagrange multiplier of a scalar symmetric traceless matrix. The equation of motion with respect to $\phi$ appears as the additional constraint, which is referred to as the simplicity condition. The simplicity condition is discussed in detail by Gielen \& Oritti ${ }^{11}$ (linear constraints) and Celada et al., ${ }^{12}$ (constraints on $\mathbb{C}$ formalism).

We note that the action $\mathcal{I}_{B F}$ does not have any topological characteristic-classes any more after implementing the constraint term. One of possible choices of the simplicity condition is to identify the form $\mathfrak{e}^{a} \wedge \mathfrak{e}^{b}$ as $\mathfrak{B}^{a b}$, and thus, a shape of the BF action coincides with the Einstein-Hilbert action. This coincidence of the shape of the action is true only for solutions of the equation of motion (onshell condition) in the classical level, and the on-shell condition cannot be simply fulfilled after quantization of the BF theory. The BF gravitational theory is complicated ${ }^{12}$ owing to this fact.

\section{Chern-Weil topological theory}

The four-dimensional Einstein-Hilbert gravitational action can be constructed using the Chern-Weil topological theory whose principal bundle consists of the co-Poincaré group ${ }^{3}$ as the structural group. The co-Poincaré principal bundle $\Theta=\left(\mathfrak{w}^{a b}, \mathfrak{S}_{c d}\right)$ is introduced into four-dimensional space-time manifold $\mathcal{M}$, where $\mathfrak{S}_{a b}:=\frac{1}{2} \varepsilon_{a b c d} \mathfrak{e}^{c} \wedge \mathfrak{e}^{d}$ is the surface form. Corresponding connection form $\mathfrak{A}_{C W}^{2}$ and curvature form $\mathfrak{F}_{C W}$ are respectively expresses as follows:

$$
\begin{aligned}
& \mathfrak{A}_{C W}:=\left(J_{a b}, P^{c d}\right) \times\left(\mathfrak{w}^{a b}, \mathfrak{S}_{c d}\right)=J_{a b} \mathfrak{w}^{a b}+P^{a b} \mathfrak{S}_{a b}, \\
& \mathfrak{F}_{C W}:=d \mathfrak{A}_{C W}+\mathfrak{A}_{C W} \wedge \mathfrak{A}_{C W}=J_{a b} \mathfrak{R}^{a b}+P^{v} d_{\mathfrak{w}} \mathfrak{S}_{a b},
\end{aligned}
$$

where $d_{\mathfrak{w}}$ is the covariant derivative with respect to $S O(1,3)$, and $J_{a b}$ and $P^{c d}$ are respectively generators of $S O(1,3)$ and the cotranslation, whose Lie algebra is expresses as

$$
\begin{gathered}
{\left[P_{a b}, P_{c d}\right]=0,} \\
{\left[J_{a b}, P_{c d}\right]=-\eta_{a c} P_{b d}+\eta_{b c} P_{a d},} \\
{\left[J_{a b}, J_{c d}\right]=-\eta_{a c} J_{b d}+\eta_{b c} J_{a d}-\eta_{b d} J_{a c}+\eta_{a d} J_{b c} .}
\end{gathered}
$$

It is proven in Kurihara $^{3}$ that the Einstein-Hilbert gravitational action without the cosmological constant can be written as $\mathfrak{L}=\operatorname{Tr}[\mathfrak{F} \wedge \mathfrak{F}]$, and thus, it has a topological invariant as Chernclasses due to the Chern-Weil theory. This result suggests that appropriate fundamental forms (phase space) of the symplectic geometry for general relativity can be identified as $(\mathfrak{w}, \mathfrak{S})$.

The base manifold $M$ is taken as Riemannian manifold with $(1+3)$ space-time dimension and the structural group is $(1+3)$ dimensional co-Poincaré group. ${ }^{3}$ The action integral of the ChernWeil topological theory can be introduced as

$$
\mathcal{I}_{C W}:=\frac{1}{4} \int_{\Sigma_{5}} \operatorname{Tr}[\mathfrak{F} \wedge \mathfrak{F}]=\frac{1}{2} \int_{\Sigma_{4}} \mathfrak{S}_{. .} \wedge \mathfrak{R}
$$

In contrast with the $\mathrm{BF}$ gravitational theory, both forms $\mathfrak{S}_{a b}$ and $\mathfrak{R}^{a b}$ re directly obtained from the principal bundle, and thus, the Einstein-Hilbert action itself preserves the characteristic class (second Chern-class), which is ensured by the Chern-Weil theory. ${ }^{3,13}$ Even though forms $\mathfrak{B}^{a b}$ and $\mathfrak{S}_{a b}$ are equivalent when the simplicity condition is required in the BF theory, the Chern-Weil gravitaional theory based on the different principal bundle with different structural group from those of the BH theory, and thus, they are completely different each other. The simplicity condition of the BF gravitational theory corresponds to the definition of the surface form in the ChernWeil topological theory, and thus, it is exact after quantization. Quantization of the Chern-Weil topological theory can be performed using gauge fixing terms with respect to the global $\operatorname{ISO}(1,3)$ and local $\operatorname{ISO}(1,3) \cdot{ }^{14}$

In contrast with the gravitational theory in a three-dimensional space-time, the Chern-Weil theory in four-dimension has the dynamical DOF after quantization. Among $10 \mathrm{DOF}$ on the symplectic fields of the vierbein and spin forms, 2 physical degrees are remaining as the dynamical DOF, corresponding to 2 spin-states of graviton. Quantization of constrained system can be performed using the Kugo-Ojima formalism. ${ }^{15}$ At first, the auxiliary, ghost and anti-ghost fields are introduced to fix the gauge and unphysical DOF in the system. Then the BRS transformations are required on all of physical and unphysical fields. At this stage, number of constraints due to the BRS transformations is the same as a total DOF in the system, and thus, there is no dynamical degree if all constraints are independent each other. In reality, as explained in Kurihara ${ }^{14}$ all constraints are not independent and there are two conserved BRS charges in the system. Therefore, the system still has two dynamical DOF after quantization. At the end, principal bundle and its structural group for three topological theories are summarized in Table 1. 
Table I A summary of topological theories.

\begin{tabular}{lll}
\hline Topological theory & Principal bundle & Structural group \\
\hline Chern-Simons & $(\mathfrak{e}, \mathfrak{w})$ & $S O(1,2)$ \\
$\mathrm{BF}$ & $(\mathfrak{B}, \mathfrak{w})$ & $S O(1,3)$ \\
Cherm-Weil & $(\mathfrak{w}, \mathfrak{S})$ & Co-Poincarè $(I, 3)$ \\
\hline
\end{tabular}

\section{Acknowledgements}

None.

\section{Conflicts of interest}

Author declares there is no conflict of interest.

\section{References}

1. Witten E. Topological quantum field theory. Communications in Mathematical Physics. 1988;117(3):353-386.

2. Zanelli J. Chern-Simons forms in gravitation theories. Classical and Quantum Gravity. 2012;29(3).

3. Kurihara Y. Characteristic classes in general relativity on a modified Poincaré curvature bundle. Journal of Mathematical Physics. 2017;58(9):1-10.

4. Hassaine M, Zanelli J. Chern-Simons (Super) Gravity. Singapore: World Scientific; 2016
5. Plebanski JF. On the separation of Einsteinian substructures. Journal of Mathematical Physics. 1977;18(12).

6. Horowitz GT. Exactly soluble diffeomorphism invariant theories. Communications in Mathematical Physics. 1989;125(3):417-437.

7. Birmingham D, Blau M, Rakowski M, et al. Topological field theory. Physics Report. 1991;209(4-5):129-340.

8. Krasnov K. Plebański formulation of general relativity: a practica introduction. General Relativity and Gravitation. 2011;43(1):1-15.

9. Girelli F, Pfeiffer H. Higher gauge theory-differential versus integral formulation. Journal of Mathematical Physics. 2004;45(10).

10. Pietri RD, Freidel L. so(4) Plebanski action and relativistic spin-foam model. Classical and Quantum Gravity. 1999;16(17).

11. Gielen S, Oriti D. Classical general relativity as BF-Plebanski theory with linear constraints. Classical and Quantum Gravity. 2010;27(18).

12. Celada M, Gonzalez D, Montesinos M. BF gravity. Classical and Quantum Gravity. 2016;33(21)

13. Chern SS. Differential geometry of fiber bundles. USA: International Congress of Mathematicians; 1952. p. 397-411.

14. Kurihara Y. General relativity as the four-dimensional Chern-Weil theory. USA: Cornell University Library; 2017. 1-41.

15. Nakanishi N, Ojima I. Covariant Operator Formalism of Gauge Theories and Quantum Gravity. Singapore: World Scientific Publishing Company; 1990. 